\title{
Linear and nonlinear optical properties of 4-nitrobenzoic acid (4-NBA) single crystals
}

\author{
K SANGEETHA $^{1, *}$, S THAMOTHARAN ${ }^{2}$, R RAMESH BABU $^{3}$ and S MADAN KUMAR ${ }^{4}$ \\ ${ }^{1}$ School of Electrical and Electronics Engineering, SASTRA Deemed University, Tirumalaisamudram, Thanjavur \\ 613 401, India \\ ${ }^{2}$ Biomolecular Crystallography Laboratory, School of Chemical and Biotechnology, SASTRA Deemed University, \\ Thanjavur 613 401, India \\ ${ }^{3}$ Crystal Growth and Thin Film Laboratory, Department of Physics, Bharathidasan University, Tiruchirappalli 620024 , \\ India \\ ${ }^{4}$ PURSE Laboratory, Mangalore University, Mangalagangotri, Mangalore 574 199, India \\ *Author for correspondence (sangeetha.proj@gmail.com; sangeetha@eee.sastra.edu)
}

MS received 5 July 2017; accepted 19 September 2017; published online 18 May 2018

\begin{abstract}
Nitrobenzoic acid (4-NBA) single crystals were studied for their linear and nonlinear optical properties. The crystals were grown by slow solvent evaporation method at room temperature. The structure and functional groups were confirmed by X-ray diffraction (XRD) and Fourier transform infrared (FTIR) spectroscopic studies. The 4-NBA crystal has an optical absorption at $263 \mathrm{~nm}$ and a wide transparency window for the visible light. Theoretical predictions for the measured optical absorption and charge transfer mechanism in 4-NBA were explained with HOMO and LUMO illustrations. The crystal is found to be birefringent and has produced an optical quality interference pattern. The optic plane of the crystal is observed along $\langle 100\rangle$ direction. The crystal exhibits nonlinear optical effects viz. reverse saturable absorption and selfdefocussing of laser beam. Considerable nonlinear refraction $\left(n_{2}\right)$ and nonlinear absorption $(\beta)$ coefficients and third-order nonlinear optical susceptibility $\left(\chi^{(3)}\right)$ were determined using Z-scan technique.
\end{abstract}

Keywords. Defect analysis; mechanical stability; UV-visible spectroscopy; refractive index; birefringence; third-order nonlinear susceptibility.

\section{Introduction}

Materials that behave in nonlinear fashion to an intense laser beam are called as 'nonlinear optical (NLO) materials'. Conversion of invisible lasers into visible lasers of high energy, self-focussing, self-defocussing, optical limiting and optical switching of laser beams are all possible today by these NLO materials. The electric field strength of laser and internal atomic fields of materials are nearly equal and so all the above facts are realized in a NLO material. Electro-optic modulation, optical imaging and parametric oscillation [1-4] are some added applications of NLO materials. Due to these growing applications of NLO materials, they are constantly attracting technological importance for a long time $[5,6]$. A material is considered to be potential for optical nonlinearity if its structure satisfies more than one of the following: (i) $\pi$ electron delocalization, (ii) lengthy conjugation, (iii) donor substitution, (iv) presence of donor-acceptor groups and (v) presence of donor-neutral-acceptor groups [5]. All of these five structural factors are able to trigger optical nonlinearity greatly.

4-Nitrobenzoic acid (4-NBA) is one such organic molecular structure with $\pi$-electrons delocalized in phenyl ring and a nitro-group attached to it. Hence, this material is chosen for linear as well as nonlinear optical studies. This material (4-NBA) is thermodynamically and chemically sensitive and hence, it crystallizes in monoclinic crystal system with three different centrosymmetric space groups $(A 2 / a$, $P 2_{1} / n$ and $C 2 / c$ ) [6-8]. There were very few reports on pure 4-NBA that includes a vibrational study (Fourier transform infrared (FTIR) and NMR) [9]. Derivatives of 4-NBA single crystals [10-15] were concentrated much compared to pure 4-NBA, so as to generate second harmonic frequency (SHF) which is another optical nonlinearity exhibited only by non-centrosymmetric structured crystals. It must be brought to the attention of the readers that except second harmonic generation (SHG), centrosymmetric crystals are capable of producing all NLO phenomena and are gaining equal importance in the field of nonlinear optics.

To the best of our knowledge, there were no systematic studies on the proper growth, linear and nonlinear optical properties of pure 4-NBA single crystals. Therefore, the present work aims to understanding the linear and nonlinear optical properties of pure 4-NBA single crystals. Linear optical properties viz. optical transparency, refractive index $\left(n_{0}\right)$ and birefringence $(\Delta n)$ and NLO properties viz. selfdefocussing, nonlinear refraction $\left(n_{\mathrm{nl}}\right)$ and nonlinear absorption $(\beta)$ are experimented in detail. In addition, third-order 


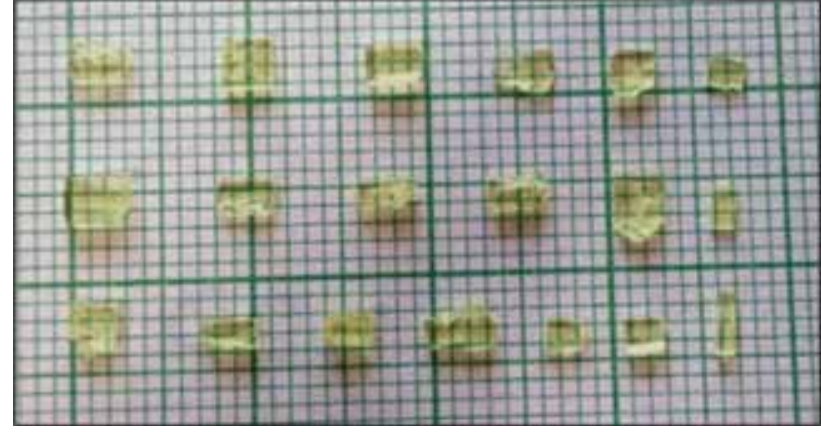

Figure 1. As-grown single crystals of 4-NBA.

NLO susceptibility $\left(\chi^{(3)}\right)$ that cause self-defocussing in the crystal is also calculated from the experimental Z-scan measurements.

\section{Experimental}

\subsection{Growth of 4-NBA single crystals}

4-NBA single crystals were grown by slow solvent evaporation method at room temperature using ethanol as solvent. Solubility of 4-NBA in ethanol is moderate that facilitated the quality of the grown crystals. Crystals were nucleated after two days and were harvested after seven days (figure 1). The crystals are observed to be rectangular in shape (platelike) with a maximum dimension of $4 \times 3 \times 0.5 \mathrm{~mm}$. The faces of the grown crystals were identified using a program 'WinXmorph' [16]. Crystallographic axes and crystal symmetry were the inputs given to the program and the predicted morphology of crystal is presented in figure 2 . The dominant faces of the crystals are $\left(\begin{array}{lll}1 & 0 & 0\end{array}\right)$ and $\left(\begin{array}{lll}-1 & 0 & 0\end{array}\right)$. In addition, some of the grown crystals were thick enough to show (001) and (010) faces.

\subsection{Characterization techniques}

The grown 4-NBA single crystals were confirmed by single crystal X-ray diffraction using Rigaku AFC12 Saturn724 + diffractometer. The vibrations of functional groups and other bonds were recorded and confirmed through FTIR spectroscopic analyses using Perkin-Elmer FTIR spectrometer in the range of 400-4000 $\mathrm{cm}^{-1}$. The structure of 4-NBA determined experimentally was used for geometry optimization in the gas and solvent phases (ethanol). The optimization was carried out using M052X/6-31+G(d) level of theory using the program Gaussian 09 [17]. The CPCM method was used to include the effect of the ethanol solvent. Further, vibrational frequencies were computed to confirm the proper convergence and found no imaginary frequencies.

The quality of the grown crystals was analysed by chemical etching technique. The crystal surface is chemically etched

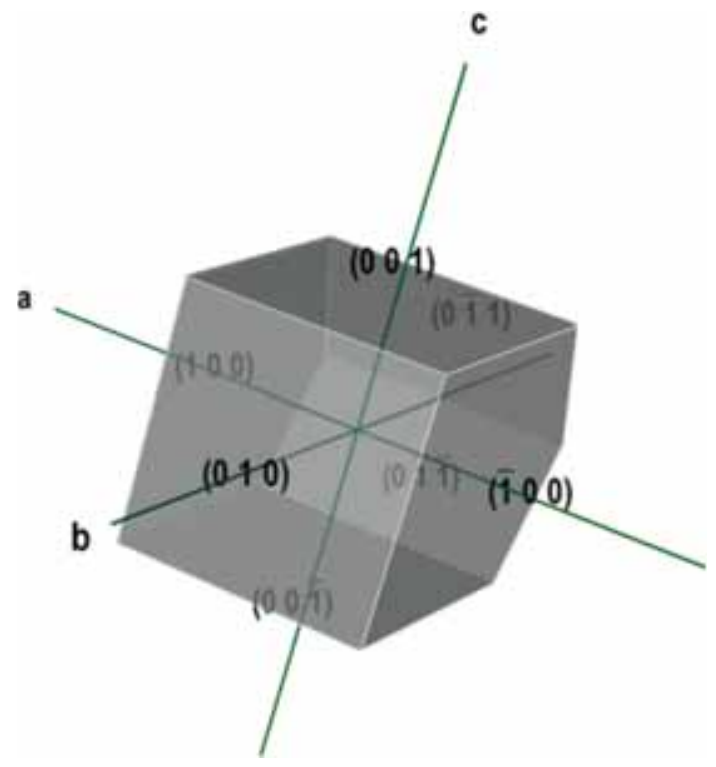

Figure 2. Morphology of 4-NBA single crystal.

with ethanol for different time durations and dried using a tissue paper. The etched dry surface of the crystal was then viewed through an optical microscope to analyse the growth mechanism and defects in the crystal. Mechanical stability is another important micro-scale property which reveals the elasticity of the crystal lattice. This micro-mechanical hardness was measured for 4-NBA using Vickers' microhardness tester (HMV-2 Shimadzu) fitted with a diamond pyramidal indenter and attached with an optical microscope. The time of indentation was $10 \mathrm{~s}$ for all the crystals. The impression of diagonals $(d)$ was measured and the microhardness $\left(H_{\mathrm{v}}\right)$ value was estimated from $H_{\mathrm{v}}=1.8544 P / d^{2}$, where $P$ is the applied load. Lambda 35 UV-visible spectrometer (190$800 \mathrm{~nm}$ ) was used to record the percentage of transmittance and absorptions of the crystal in UV-visible region.

The refractive index $\left(n_{0}\right)$ and birefringence $(\Delta n)$ were calculated using the formulae, $n_{0}=\tan \theta_{\mathrm{p}}$ and $\Delta n=$ $(\lambda / \pi L) \sin ^{-1}\left(I / I_{0}\right)^{1 / 2}$, respectively. The values of $\theta_{\mathrm{p}}, I$ and $I_{0}$ were collected from the experiment using diode laser source of wavelength $(\lambda) 650 \mathrm{~nm}$ (power: $20 \mathrm{~mW}$ ). The $\theta_{\mathrm{p}}$ is the polarizing angle at which the reflected light intensity from the sample surface becomes zero and transmitted light through the sample is fully polarized, $L$ is the thickness of the sample; $I_{0}$ is the intensity at the detector with sample when the polarizers are parallel and $I$ is the intensity at the detector with sample when the polarizers are crossed. The intensity $I_{0}$ includes the transmission losses of the crystal and polarizers. Hence, when the polarizers are crossed, the measured $I$ gives exactly the intensity due to birefringence of the crystal [18].

Z-scan is an intensity measurement technique in which sample in scanned before and after the focus of a convex lens. This is carried out to observe the nonlinear effects produced by the crystal. The nonlinearity changes the intensity of the laser beam at the detector and so intensity pattern is 
observed through the sample transit. This intensity variation is useful to calculate nonlinear refractive index $\left(n_{\mathrm{nl}}\right)$, nonlinear absorption $(\beta)$ and third-order NLO susceptibility $\left(\chi^{(3)}\right)$. A continuous wave $(\mathrm{CW})$ diode laser of wavelength $650 \mathrm{~nm}$ is used as source.

\section{Results and discussion}

\subsection{Single crystal XRD and FTIR spectroscopic studies}

The unit cell dimensions and space group of the grown crystal were measured using single crystal X-ray diffraction (XRD). The measured reflections indicate the 4-NBA crystallized in monoclinic crystal system with centrosymmetric space group $C 2 / c$. Unit cell dimensions measured are $a=21.2833(19)$ $\AA, b=5.0360(7) \AA, c=12.9026(11) \AA, \beta=96.751(7)^{\circ}$ and $V=1373.3(3) \AA^{3}$. Single crystal XRD data are in good agreement with the reported values [8].

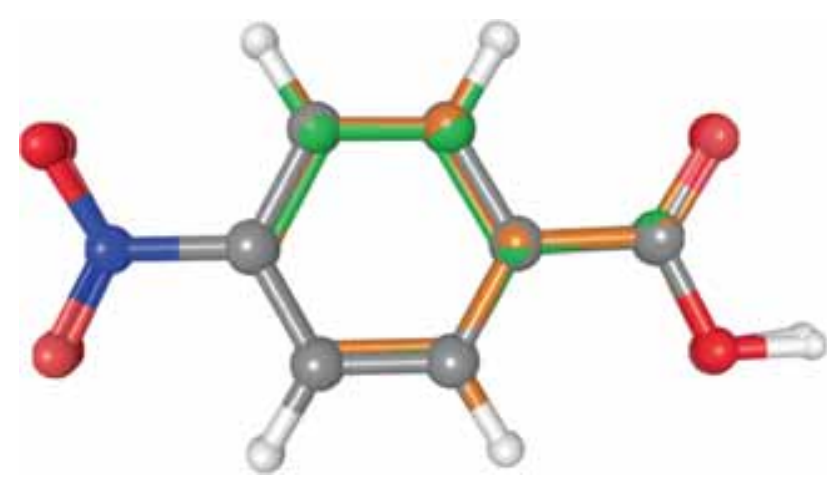

Figure 3. Superimposed structures of 4-NBA determined experimentally (grey), optimized in solution (orange) and gas phases (green).

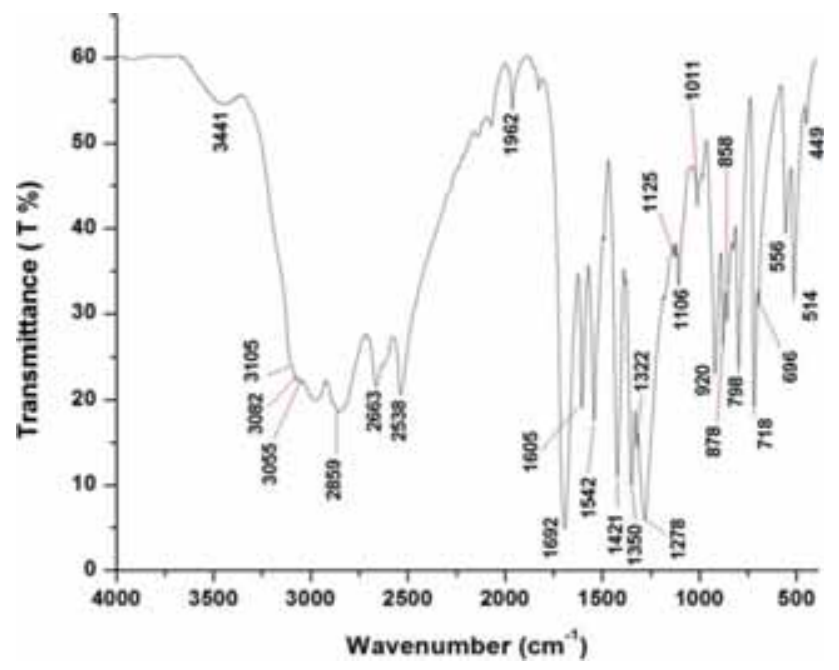

Figure 4. FTIR spectrum of 4-NBA.
Table 1. FTIR vibrations at different frequencies and their corresponding bond assignments.

\begin{tabular}{lc}
\hline Absorbed frequencies & Corresponding assignments \\
\hline 3441 & OH stretching \\
3105,3082 and 3055 & Aromatic C-H stretching \\
1962 & $\mathrm{C}-\mathrm{C}-\mathrm{O}$ bending \\
1692 & Asymmetric $\mathrm{NO}_{2}$ stretching \\
1542 & Asymmetric COO \\
1421 & $\mathrm{v}$ s $(\mathrm{COO})$ \\
1350 & Symmetric $\mathrm{NO}_{2}$ \\
1322 & $\mathrm{C}-\mathrm{C}$ \\
1278 & $\mathrm{v}(\mathrm{C}-\mathrm{OH})$ \\
1125 & $\mathrm{C}-\mathrm{C}$ \\
1106 & $\beta(\mathrm{CH})$ \\
1011 & $\beta(\mathrm{CH})$ \\
920 & $\gamma(\mathrm{CH})$ \\
878 & $\left.\beta \mathrm{s}(\mathrm{NO})_{2}\right)$ \\
858 & $\beta \mathrm{s}(\mathrm{COO})$ \\
798 & $\gamma \mathrm{s}(\mathrm{COO})$ \\
718 & $\left.\gamma \mathrm{s}(\mathrm{NO})_{2}\right)$ \\
696 & $\gamma(\mathrm{CH})$ \\
556 & $\beta$ as $(\mathrm{COO})$ \\
514 & $\beta$ as $(\mathrm{NO} 2)$ \\
449 & $\varphi(\mathrm{CC})$ \\
\hline
\end{tabular}

The optimized structures (section 2.2) were superimposed with the X-ray structure as shown in figure 3. There exists a minimum deviation $(0.1003 \AA)$ between experimentally determined structure and optimized structure in gas phase. Negligible deviation was noted between structures optimized in gas and solution phases. Furthermore, the time-dependent DFT calculation was carried out in ethanol solvent at the M052X/6-31+G(d) level theory. The choice of the basis set was made based on our previous studies [19,20].

Figure 4 represents the FTIR spectrum of 4-NBA. It is observed that the functional groups $\left(\mathrm{NO}_{2}\right.$ and $\left.\mathrm{COOH}\right)$ marked significant absorptions at corresponding frequencies (table 1). Different absorption frequencies recorded in the spectrum were identified and were assigned (table 1).

\subsection{Defect analysis}

Defects play a major role in the reliability of physical properties and efficiency of crystals in device applications. Therefore, it is essential to analyse the crystalline quality before analysing the optical properties. Chemical etching is an effective method to analyse the defects in the crystals. The chemical etching is an inverse process of growth and reveals the type of defects and defect density through etch pits and etch pit density, respectively. Figure 5a shows the surface of as-grown crystal viewed through an optical microscope $(100 \times$ resolution). Elliptical growth patterns are formed after etching the surface of as-grown crystal for 2 and 


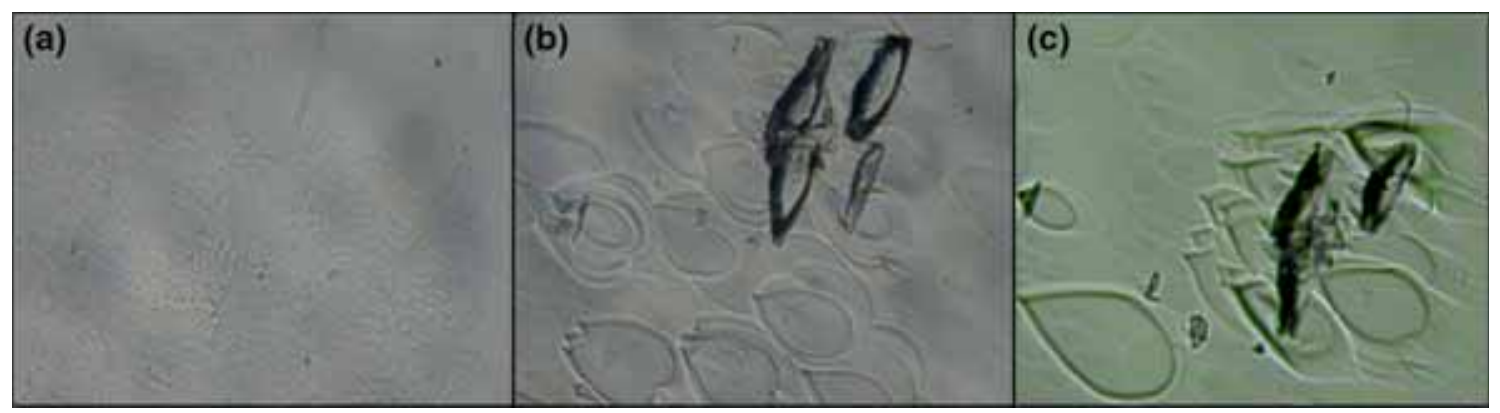

Figure 5. Etch patterns of 4-NBA at (a) 0 (as-grown), (b) 2 and (c) $4 \mathrm{~min}$.

4 min (figure $5 \mathrm{~b}$ and c) using ethanol as etchant. The elliptical growth pits formed are from screw dislocations. Layer step sequence is shown in figure $5 \mathrm{~b}$ and is the manifestation of two-dimensional layer growth mechanism. Due to the rapid growth of crystals, the etch pits are formed quickly and are overlapped. On increasing the etching time from 2 to $4 \mathrm{~min}$, the etch pits grow in size and decrease in number (figure $5 \mathrm{c}$ ). This shows the decreased defects in the internal layers of the crystal.

\subsection{Mechanical stability}

Micro-mechanical hardness is different from macro-hardness of any material and is important in device fabrication. Type of interactions between the molecules along different crystal orientations and nature of defects in the crystals greatly decide the micro-mechanical stability. $H_{\mathrm{v}}$ refers to the Vickers' hardness number which varies with applied load and work-hardening effects of the material. Figure 6a shows the Vickers' microhardness of 4-NBA with respect to load. The $H_{\mathrm{v}}$ decreases on increasing the load and above $100 \mathrm{~g}$, the crystal started cracking around the indentation mark. The work-hardening exponent $(n)$ calculated from figure $6 \mathrm{~b}$ shows that the crystal belongs to soft category $(n=1.55)$ [21]. Hardness of the crystal at $10 \mathrm{~g}$ is around $32 \mathrm{~kg} \mathrm{~mm}^{-2}$ and this is higher than the Vickers' micro-hardness of Guanidinium 4-nitrobenzoate [15] and m-nitrobenzoic acid m-nitroaniline p-xylene [22] single crystals.

\subsection{UV-visible spectroscopic study}

Optical transmittance of 4-NBA was analysed under UVvisible irradiation. Linear optical absorption occurs in a molecule, when there is an electronic transition between the bonding, non-bonding and anti-bonding orbital energy levels. Figure 7a shows the optical absorption spectrum of 4-NBA single crystal. Theoretically predicted HOMO to LUMO transition is the reason for the observed absorption at $263 \mathrm{~nm}$. The excitation energies, oscillator strengths $(f)$ and the electron configuration for some of the excited states are presented in table 2. At HOMO, the electrons were localized in the phenyl ring of 4-NBA and when excited to LUMO, where electrons
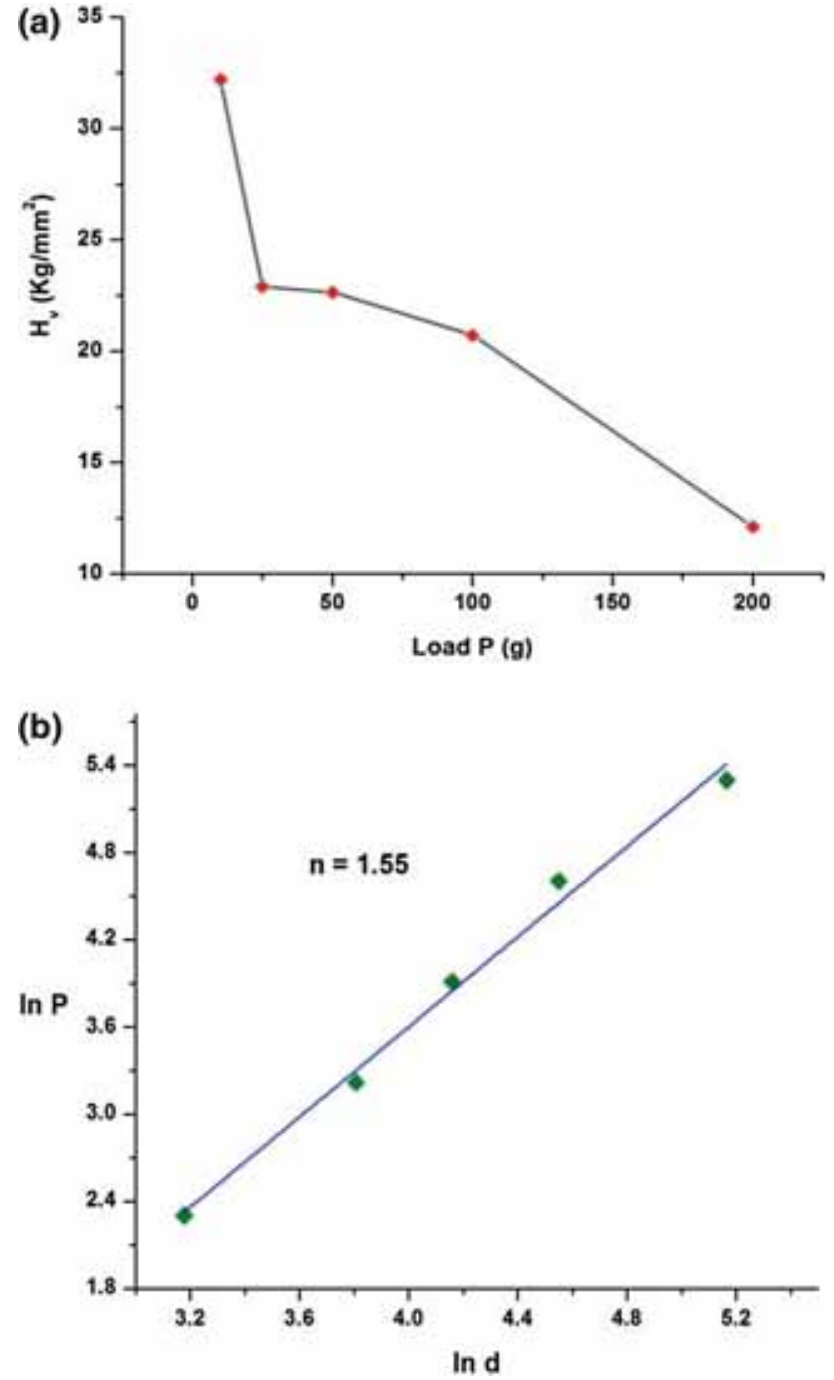

Figure 6. (a) Vickers' microhardness and (b) plot of $\ln d v s . \ln P$ of 4-NBA single crystal.

are distributed throughout the molecule as shown in figure $7 \mathrm{~b}$. Thus, charge transfer is taking place in the molecule from phenyl ring to the substitutions as illustrated. The energy band gap between HOMO and LUMO is calculated to be $7.21 \mathrm{eV}$ 


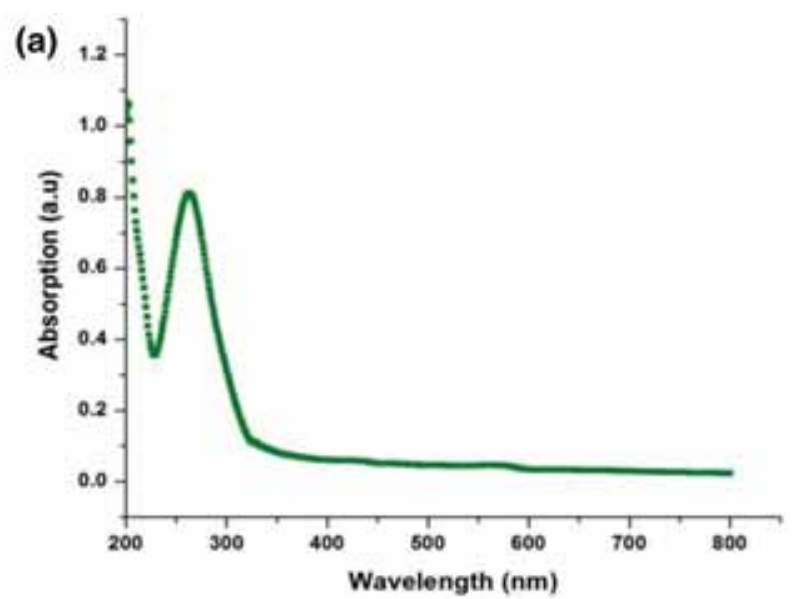

(b)
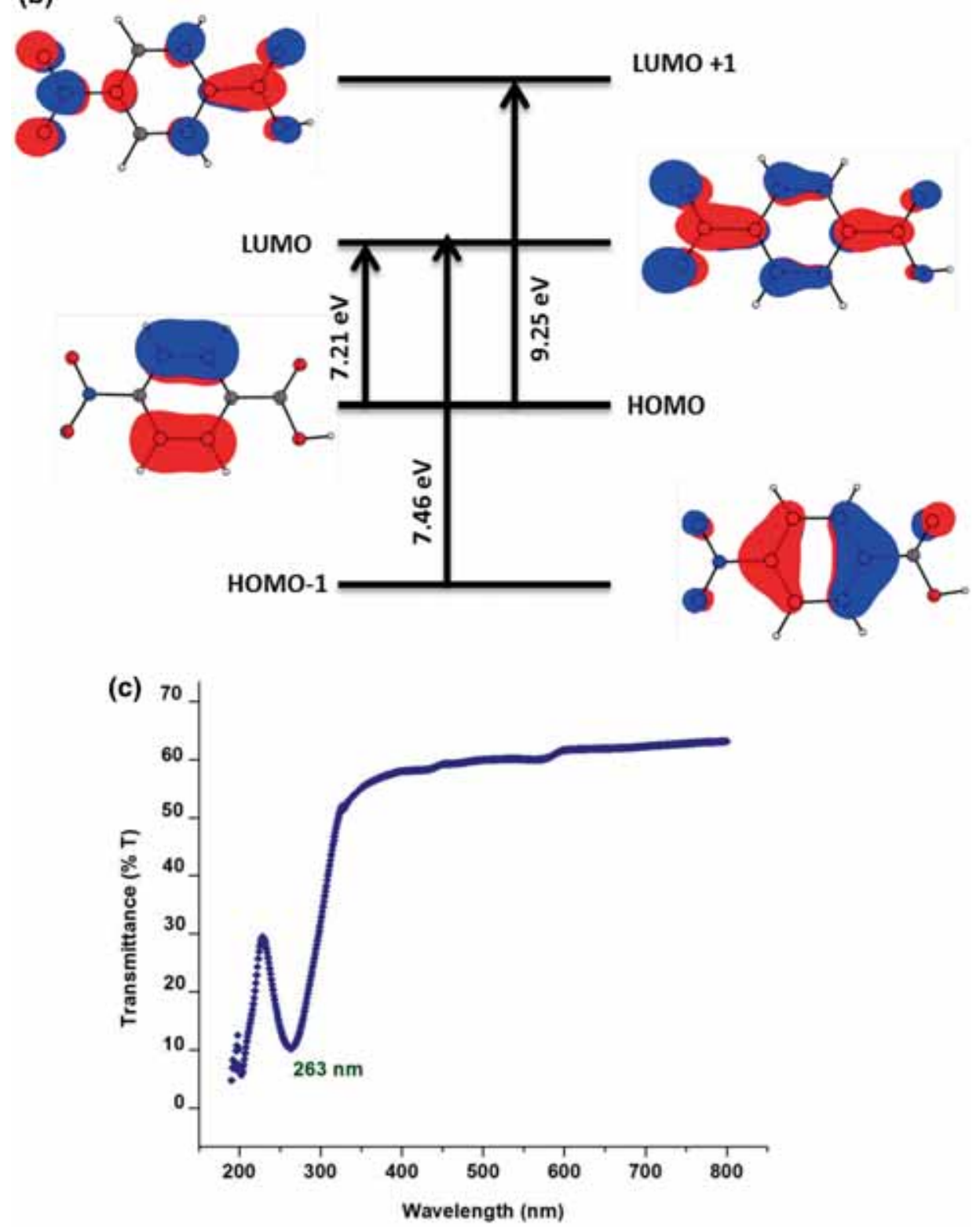

Figure 7. (a) Optical absorption of 4-NBA single crystal. (b) Electron distributions in HOMO and LUMO. (c) Optical transmittance of 4-NBA single crystal. 
Table 2. Z-scan experiment details and results for 4-NBA single crystal.

\begin{tabular}{lc}
\hline Laser parameters & \\
Laser & Diode laser \\
Wavelength & $650 \mathrm{~nm}$ \\
Power & $20 \mathrm{~mW}$ \\
Experimental details & \\
Sample thickness, $L$ & $5 \times 10^{-4} \mathrm{~m}$ \\
Focal length of lens, $f$ & $8 \times 10^{-2} \mathrm{~m}$ \\
Beam radius at aperture, $\omega_{\mathrm{a}}$ & $7.5 \times 10^{-3} \mathrm{~m}$ \\
Intensity of laser at focus, $I_{0}$ & $0.118 \mathrm{MW}^{-3} \mathrm{~m}$ \\
Radius of beam at aperture, $r_{\mathrm{a}}($ closed aperture) & $4 \times 10^{-3} \mathrm{~m}$ \\
Results & \\
Nonlinear refractive index, $n_{\mathrm{nl}}$ & $1.44 \times 10^{-6} \mathrm{~cm}^{2} \mathrm{~W}^{-1}$ \\
Nonlinear absorption coefficient, $\beta$ & $0.41626 \mathrm{~cm} \mathrm{~W}^{-1}$ \\
Real part of $\chi^{(3)}$ & $3.9524 \times 10^{-5} \mathrm{esu}$ \\
Imaginary part of $\chi^{(3)}$ & $5.9128 \times 10^{-5} \mathrm{esu}$ \\
Third-order nonlinear susceptibility, $\chi^{(3)}$ & $7.1122 \times 10^{-5} \mathrm{esu}$ \\
\hline
\end{tabular}

indicating that 4-NBA is a dielectric material. In the view of transparency (figure 7c), the crystal is widely transparent to all visible radiations $(60 \%)$.

\subsection{Refractive index and birefringence}

Refractive index along $\langle 100\rangle$ orientation of the crystal is measured to be 1.22 for a diode laser of wavelength $650 \mathrm{~nm}$. Birefringence is yet another linear optical property which plays a major role in determining the optic axes, angle between the optic axes and optic sign of the biaxial crystal. 4-NBA single crystal (monoclinic) is biaxial with two optic axes and three refractive indices.

The birefringence can be viewed through a plane of the crystal, which is perpendicular to the optic axis. This plane allows the ordinary ray (OR) and extra-ordinary ray (ER) to travel in same direction, but with different velocities. Therefore, the plane facilitates the rays to interfere and produce birefringence pattern. The $\left(\begin{array}{lll}1 & 0 & 0\end{array}\right)$ crystal face of 4-NBA may be the optic plane, which is perpendicular to the optic axis of the crystal. Figure 8 shows the birefringence pattern of 4-NBA single crystal and the birefringence value is measured to be 0.04 . Birefringence technique is an excellent tool to project the optical quality of the crystal. The continuous bright and dark fringes in the birefringence pattern represent good optical quality of the crystal.

\subsection{Third-order nonlinear optical susceptibility}

3.6a Z-scan technique: Optical nonlinearity in the crystal is triggered by irradiating a $20 \mathrm{~mW} \mathrm{CW}$ diode laser of wavelength $(\lambda) 650 \mathrm{~nm}$. Whenever a laser beam falls on a crystal, spatial distribution of temperature at the laser spot on the crystal is produced [23]. This is due to the localized absorption of the tightly focussed Gaussian laser beam

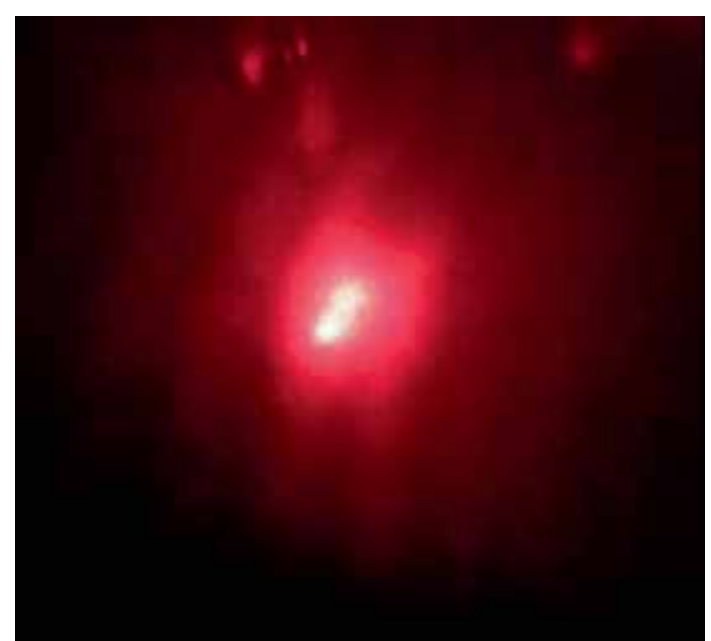

Figure 8. Birefringence pattern of 4-NBA single crystal.

propagating through the crystal medium. Intensity of the laser beam is high at a spot centre and decreases outwards from the centre as shown in figure 9a. Hence, a spatial variation of refractive index is produced (figure $9 \mathrm{~b}$ ), which acts as thermal lens resulting in self-defocussing/self-focussing of the propagating beam. Crystals are brilliant in varying the refractive indices. (1) Some crystals offer a high refractive index at the centre of the spot and decreases concentrically outwards (negative nonlinear refraction). (2) Exactly opposite are some other crystals that offer a low refractive index at the centre and it increases when moved outwards concentrically (positive nonlinear refraction). Therefore, (1) crystals defocus and (2) focus the laser beam, respectively. The variation in refractive indices, self-focussing and self-defocussing behaviours can be identified in Z-scan technique. This knowledge about the 


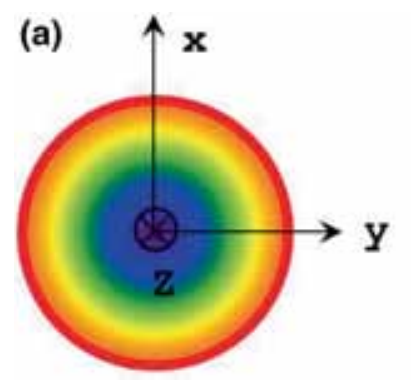

(b)

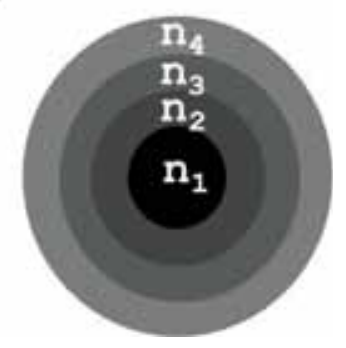

Figure 9. (a) Gaussian laser beam and (b) concentric refractive index changes at laser-irradiated crystal spot.

crystals is of great importance in optical limiting and optical switching applications [24].

$\mathrm{Z}$-scan is an intensity measurement technique in which a crystal is scanned across $Z$-axis through a small distance and observed for intensity variation. The intensity variation at the output can be attributed to the optical nonlinearity/selffocussing/self-defocussing behaviours of the crystal. Z-scan is operated in two modes: open aperture $(\mathrm{OA})$ mode and closed aperture (CA) mode to determine the values of $n_{\mathrm{nl}}$ and $\beta$, respectively. In OA mode, no aperture is placed in front of photodiode and hence, all light transmitting through the sample is determined. Thus, the detected intensity is for the nonlinear absorption $(\beta)$ of the sample. In CA mode, a small aperture is placed at a distance in front of the photodiode and only the central part of transmitted light through the aperture falls on the photodiode when the sample is scanned along $Z$-axis. The intensity varies with $Z$ position and produces a peak-valley or valley-peak pattern. The sign and magnitude of the nonlinear refraction index coefficient can be determined from the result [25].

4-NBA single crystal of thickness $0.5 \mathrm{~mm}$ was used to measure the nonlinear refractive index $\left(n_{\mathrm{nl}}\right)$, nonlinear absorption coefficient $(\beta)$ and third-order NLO susceptibility $\left(\chi^{(3)}\right)$ using Z-scan technique. A lens of $8 \mathrm{~cm}$ focal length $(f)$ was used to focus the laser beam. Laser beam is allowed to pass through the crystal. In CA mode, the transmitted light through the aperture placed in the far field was detected by a photo detector attached to a digital power meter. In OA mode, the aperture is replaced by a lens to focus the entire transmitted light through the sample.

3.6b Mathematical descriptions: The magnitude of the phase shift $\left(\Delta \varphi_{0}\right)$ can be determined from the change in normalized transmittance between peak and valley using the relation [26],

$$
\Delta \varphi_{0}=\frac{\Delta T_{\mathrm{pv}}}{0.406(1-S)^{0.25}}
$$

Where $\Delta T_{\mathrm{pv}}$ is the difference in transmittance between peak and valley, $S$ is the aperture linear transmittance and it can be
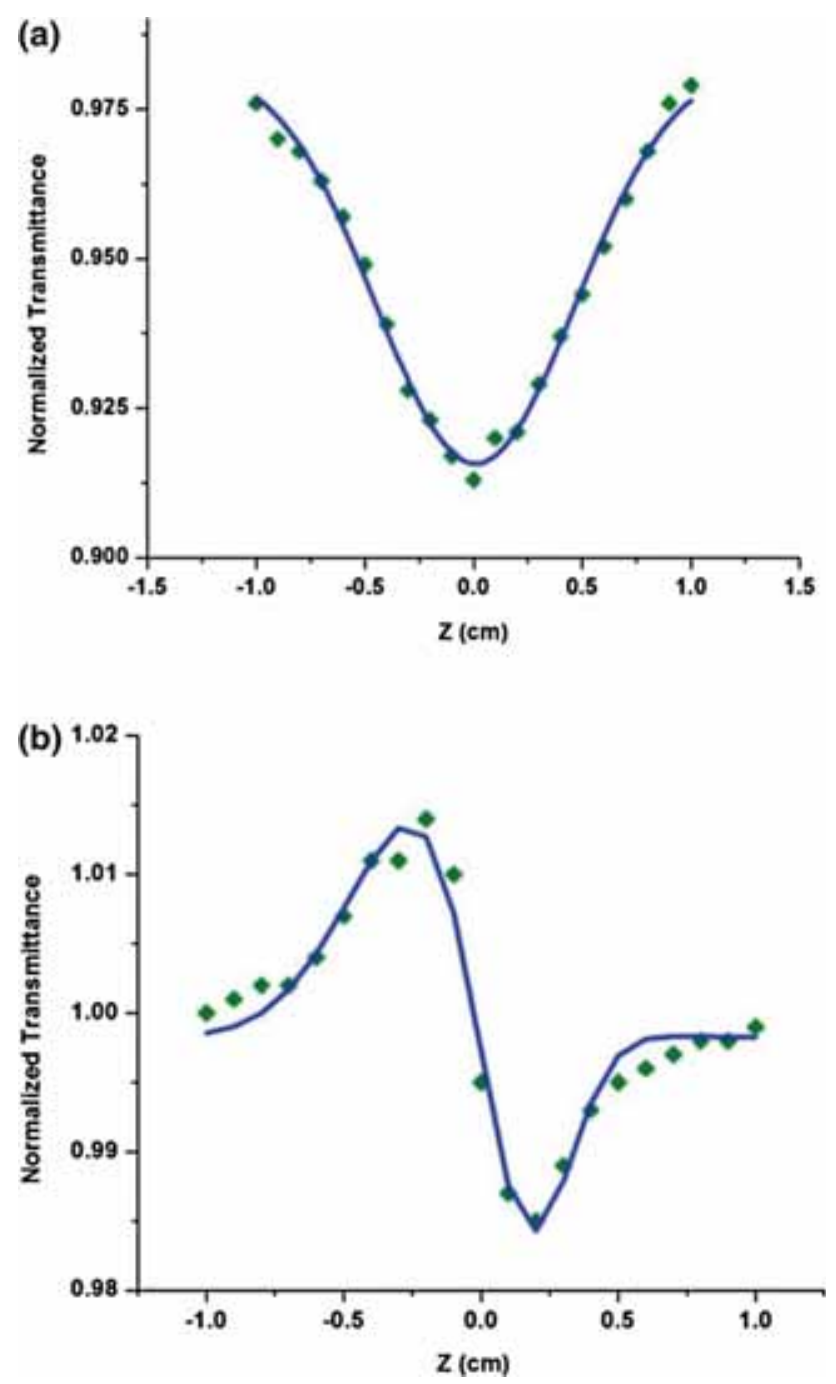

Figure 10. Z-scan normalized transmittance of 4-NBA single crystal for (a) OA and (b) CA modes.

calculated using the relation,

$$
S=1-e^{\left(-2 r_{\mathrm{a}}^{2} / \omega_{\mathrm{a}}^{2}\right)},
$$

where $r_{\mathrm{a}}$ is the aperture radius and $\omega_{\mathrm{a}}$ is the beam radius at aperture. The nonlinear refractive index, $n_{2}$, can be determined using,

$$
n_{\mathrm{nl}}=\frac{\Delta \varphi_{0}}{K I_{0} L_{\mathrm{eff}}},
$$

where $K=2 \pi / \lambda, \lambda$ is wavelength of laser, $I_{0}$ is the intensity of laser beam at focus $(Z=0), L_{\text {eff }}=[1-\exp (-\alpha L) / \alpha]$, where $\alpha$ is the linear absorption coefficient and $L$ is the thickness of the sample. The pure nonlinear refractive index $n_{\mathrm{nl}}$ is obtained by dividing the CA data by the OA data [27]. The nonlinear absorption coefficient $\beta$ is calculated from the OA 
Table 3. UV-Vis excitation energies of 4NBA in ethanol solvent. TD-DFT calculation was performed using M052X/6-31+G(d) level of theory.

\begin{tabular}{lccc}
\hline Excitation energy $(\mathrm{nm})$ & Oscillator strength, $f$ & Main configuration & Expt. (nm) \\
\hline 279 & 0.0003 & $\mathrm{H}-5 \rightarrow \mathrm{L}(79 \%)$ & \\
& & $\mathrm{H}-5 \rightarrow \mathrm{L}+1(18 \%)$ & \\
267 & 0.0518 & $\mathrm{H} \rightarrow \mathrm{L}(93 \%)$ & 263 \\
249 & 0.0006 & $\mathrm{H}-2 \rightarrow \mathrm{L}(56 \%)$ & \\
248 & 0.4733 & $\mathrm{H}-2 \rightarrow \mathrm{L}+1(33 \%)$ & \\
& & $\mathrm{H}-1 \rightarrow \mathrm{L}(96 \%)$ & \\
\hline
\end{tabular}

Z-scan data i.e.,

$$
\beta=\frac{2 \sqrt{ } 2 \Delta T}{I_{0} L_{\mathrm{eff}}},
$$

where $\Delta T$ is the normalized transmittance of the sample when at position $Z$. Experimentally determined nonlinear refractive index $n_{\mathrm{nl}}$ and nonlinear absorption coefficient $\beta$ can be used to find the real and imaginary part of the third-order NLO susceptibility according to the following relations [28],

$$
\begin{aligned}
& \operatorname{Re} \chi^{(3)}(\mathrm{esu})=10^{-4} \frac{\varepsilon_{0} c^{2} n_{0}^{2} n_{\mathrm{nl}}}{\pi}, \\
& \operatorname{Im} \chi^{(3)}(\mathrm{esu})=10^{-2} \frac{\varepsilon_{0} c^{2} n_{0}^{2} \lambda \beta}{4 \pi^{2}},
\end{aligned}
$$

where $\varepsilon_{0}$ is the vacuum permittivity, $n_{0}$ is the linear refractive index of the sample and $c$ is the velocity of light in a vacuum.

The absolute value of the third-order NLO susceptibility is given by the relation,

$$
\left|\chi^{(3)}\right|=\left[\left(\operatorname{Re} \chi^{(3)}\right)^{2}+\left(\operatorname{Im} \chi^{(3)}\right)^{2}\right]^{1 / 2} .
$$

3.6c Observations: Figure 10a and b shows the Z-scan curves of 4-NBA single crystal. The experimental details and the result of Z-scan for 4-NBA crystal are shown in table 3. The results tabulated correspond to the parameters of the laser source.

It is observed from figure $10 \mathrm{a}$ (OA mode) that the curve is symmetric about the focus $(Z=0)$ at which the measured transmittance forms a valley and such a behaviour is known as Reverse saturable absorption (RSA) of the crystal. The RSA is a positive type of absorption nonlinearity in which the absorption increases at focus (at high intensity) and decreases away from the focus (at low intensities). Figure 10b indicates a prefocal transmittance maximum (peak) followed by a post-focal transmittance minimum (valley) in the scanned range. This indicates that the crystal has a negative nonlinear refraction $\left(n_{\mathrm{nl}}\right)$, in which the refractive index is high at the centre and decreases outwards concentrically (figure 9b). Thus, 4-NBA crystal self-defocusses the laser beam.
From equation (7), it is clear that real and imaginary parts contribute to the third-order NLO susceptibility $\left(\chi^{(3)}\right)$. The contribution from imaginary part is observed (table 3 ) to be larger than the real part specifying the dominance of nonlinear absorption over nonlinear refraction. Therefore, there exists a possibility of decrease in the NLO susceptibility $\left(\chi^{(3)}\right)$ value when the laser intensity is increased.

\section{Conclusions}

Structural, linear and NLO properties of 4-NBA single crystals were investigated systematically. The grown crystals had minimum defects in the internal layers compared to the surface layers of the crystal. This has resulted in a perfect elastic lattice arrangement and comparatively a high micromechanical stability. 4-NBA crystal has moderate percentage of optical transmittance (60\%) and is widely transparent to visible light. The crystal is observed to be birefringent exhibiting continuous bright and dark interference fringes along $\langle 100\rangle$ direction. Z-scan studies revealed significant nonlinear effects in 4-NBA crystal. The crystal has positive nonlinear absorption and negative nonlinear refraction. The nonlinear absorption $(\beta)$ of the crystal is dominant over nonlinear refraction $\left(n_{\mathrm{nl}}\right)$ for the incident intensity at focus. The RSA exhibited by 4-NBA indicates its effectual role in opticallimiting applications.

\section{Acknowledgements}

The author ST thankfully acknowledges the DST-SERB (SB/YS/LS-19/2014) and Prof TRR grant for financial support.

\section{References}

[1] Zhao P, Wang Z, Chen J, Zhou Y and Zhang F 2017 Optic. Mater. 6698

[2] Sreeramulu Valligatla, Krishna Kanta Haldar, Amitava Patra and Narayana Rao Desai 2016 Optic. Laser Technol. 8487 
[3] Ekbote A, Patil P S, Maidur S R, Chia T and Quah C K 2017 Dyes Pigments 139720

[4] Henari F Z and Patil P S 2014 Opt. Photonics J. 4182

[5] Nalwa H S and Miyata S 1997 Nonlinear optics of organic molecules and polymers (United States of America: CRC Press, Inc.)

[6] Masahiko Tonogaki, Takashi Kawata and Shigeru Oi-Iba 1993 Acta Cryst. B49 1031

[7] Bolte M 2009 Private communication to the CCDC Cambridge, England (CSD Reference Code: NBZOAC11)

[8] Thakur T S and Singh S S 2015 Cryst. Growth Des. 153280

[9] Samsonowicz M, Swislocka R, Regulska E and Lewandowski W 2007 Int. J. Quantum Chem. 107480

[10] Roy S M, Sudarsanakumar M R, Dhanya V S, Suma S and Prathapachandra Kurup M R 2014 J. Korean Chem. Soc. 58 258

[11] Hatem M Titi, Goutam Nandi, Ranjit Thakuria and Israel Goldberg 2015 Inorg. Chim. Acta $\mathbf{4 2 6} 55$

[12] Samsonowicz M, Regulska E, Wislocka R S and Lewandowski W 2013 Spectrochim. Acta A $\mathbf{1 0 3} 456$

[13] Quah C K, Jebas S R and Fun H-K 2008 Acta Cryst. E64 o1878

[14] Chandramohan A et al 2014 Experiment 221537

[15] Arumanayagam T and Murugakoothan P 2013 J. Cryst. Growth $\mathbf{3 6 2} 304$
[16] Kaminsky W 2005 J. Appl. Cryst. 38566

[17] Frisch M J et al 2013 Gaussian 09 revision D.01 (Wallingford, CT: Gaussian Inc.)

[18] Nasr A M 2007 Int. J. Mater. Sci. 2103

[19] Venkatesan P, Rajakannan V, Venkataramanan N S, Ilangovan A, Sundius T and Thamotharan S 2016 J. Mol. Struct. 1119 259

[20] Mani Udayakumar et al 2017 J. Mol. Struct. 1133510

[21] Senthil Murugan G and Ramasamy P 2016 Optik 127 4007

[22] Jagdish P, Rajesh N P and Natarajan S 2010 J. Miner. Mater. Charact. Eng. 9471

[23] Gupte S S, Marcano A, Pradhan R D, Desai C F and Melikechi N 2001 J. Appl. Phys. 894939

[24] Praveen P A, Ramesh Babu R and Ramamurthi K 2017 Spectrochim. Acta A 173800

[25] Srinivasan P, Nooraldeen A Y, Kanagasekaran T, Dhinaa A N, Palanisamy P K and Gopalakrishnan R 2008 Laser Phys. 18 790

[26] Mathews S J, Chaitanya Kumar S, Giribabu L and Venugopal Rao S 2007 Mat. Lett. 614426

[27] Sheik-Bahae M, Said A A, Wei T H, Hagan D J and Van Stryland E W 1990 IEEE J. Quantum Electron 26760

[28] Cassano T, Tommasi R, Ferrara M, Babudri F, Farinola G M and Naso F 2001 Chem. Phys. 272111 